\title{
Performance Evaluation of Variant Error Correction Schemes in Terms of Extended Coding Rates \& BER for OFDM Based Wireless Systems
}

\author{
Md. Nurul Mustafa, Mohammad Jahangir Alam, Nur Sakibul Huda \\ Department of Computer Science and Engineering (CSE), Southern University Bangladesh, Chittagong, Bangladesh \\ Email address: \\ nurul.mustafa@southern.edu.bd (Md. N. Mustafa), jahangir@southern.edu.bd (M. J. Alam), jetsakib@yahoo.com (N. S. Huda)
}

To cite this article:

Md. Nurul Mustafa, Mohammad Jahangir Alam, Nur Sakibul Huda. Performance Evaluation of Variant Error Correction Schemes in Terms of Extended Coding Rates \& BER for OFDM Based Wireless Systems. International Journal of Wireless Communications and Mobile Computing. Vol. 4, No. 1, 2016, pp. 7-11. doi: 10.11648/j.wcmc.20160401.12

\begin{abstract}
Modern OFDM systems provide effective spectral usage by allowing overlapping in the frequency domain. Moreover, it is highly resistant to multipath delay spread. The suppression of inter-symbol interference (ISI) is one of the top features of OFDM. It also facilitates mobile bandwidth allocation and may increase the capacity in terms of number of users. Even though the presence of OFDM's built in error preventing mechanism; error tends to occur that averts delivery of proper signal. In this work we evaluated and adapted ways of fine-tuning for different error correction methods so that they may bring positive impact on communication systems who are developing and using OFDM. Here the performance of different known error correcting techniques for OFDM systems have been analyzed after extending and puncturing feature is applied on them. Simulations are performed in well-known simulator MATLAB to evaluate the modified techniques for different channel conditions. As the advantage of OFDM based systems are mainly the robustness to channel impairments and narrow-band interference; thus added error reduction models will improve significant efficiency of systems based on OFDM.
\end{abstract}

Keywords: Extended RS Code, AWGN, ISI, BER, Extended Convolution Code, Extended LB Code, FFT, IFFT

\section{Introduction}

Wireless communication, as the name suggests [1], is wireless way of transmitting information from one place to another, is replacing most of the wired transmission of today's world. Research in the field of wireless communication is still a hot topic to discover new possibilities. The goal of every research in this topic is to find more effective communication methods. Wireless communication helped the user to move freely without worrying about transfer of data. It dramatically changed the concept of information transfer in homes and in offices.

The basic building blocks of a typical wireless communication system [2], are encoder, channel coder, modulator, demodulator, and channel decoder. The signal is first converted to digital data and then source encoded. Source encoding reduces the amount of the data present in the signal to reduce the bandwidth required to transmit the associated data. Then the data proceeds to channel encoder block, which is responsible for adding extra bits in the data to help correcting errors inflicted to the data due to fading and noise. Our work in this paper contains three techniques for channel encoding that will be explained in later chapters. The modulator modulates the message signal on the transmission frequency so that the signal is ready for transmission.

Signal when transmitted, through wireless channel, faces multiple problems [3]. One major problem faced by the signal is fading. Fading can be caused by natural weather disturbances, such as rainfall, snow, fog, hail and extremely cold air over a warm earth. Fading can also be created by manmade disturbances, such as irrigation, or from multiple paths. All these factors introduce errors into the transmitted data.

When received at the receiver the signal is added with noise, since receiver antenna is designed to receive any signal present within a certain frequency range and noise is also present in that range. Now that data is passed to the demodulator whose job is convert the signal back from the carrier frequency to its normal form. After that the channel decoder helps to recover original signal from the degraded signal due to channel fading and noise. This is done by using the redundant bits that were added by the channel encoder. 
This signal after recovery is passed to the source decoder, which converts the signal back to its original form.

\section{The Problem in Details}

OFDMis a technique, for transmission of data stream over a number of sub-carriers. In OFDM, a high rate bit stream is divided into bit streams of lower rate and each of them are modulated over one of the orthogonal subcarriers [1]. In a single carrier system a single fade can cause the entire link to fail while in a multi carrier system only a few bits will be disturbed and they can be corrected by applying error correction codes.

OFDM overcomes the problem of inter-symbol interferenceby transmitting a number of narrowband subcarriers together with a guard interval [4]. But this gives rise to another problem that all subcarriers will arrive at the receiver with different amplitudes. Some carriers may be detected without error but the errors will be distributed among the few subcarriers with small amplitude.

Channel coding can be used across the subcarriers to correct the errors of weak subcarriers. In OFDM systems error correction has a significant role since OFDM along with error correcting techniques help to deal with fading channels. Error correction helps in recovery of faded information by providing a relation between information and transmitted code such that errors occurring within the channel can be removed at the receiver. A lot of such techniques for error correction are given till date. In this work some of these techniques are considered, modified to an extent \& implemented for OFDM system and then a comparison between them are made for best outcome.

\section{Channel Coding}

Channel codingis basically from the class of signal transformations designed to improve the communication performance by enabling the transmitted signal to better resist the effects of various channel impairments such as noise fading and jamming [5]. The goal of channel coding is to improve the bit error rate (BER) performance of power limited and/or band limited channels by adding redundancy to the transmitted data. The three channel coding schemes [13], that are modified to achieve objective of this work are described in the following section:

\subsection{Linear Block Coding}

A block code is defined as a code in which $\mathrm{k}$ symbols are input and $\mathrm{n}$ symbols are output and is denoted as a (n, k) code[5]. If the input is $\mathrm{k}$ symbols, then there are $2 \mathrm{k}$ distinct messages. For each $\mathrm{k}$ input symbols output is $\mathrm{n}$ symbols known as a codeword where $n$ is greater than $k$. Figure 1 shows input and output of a block code and the size of codeword formed.

A block code of length $\mathrm{n}$ with 2 kcodewords is called a linear $(\mathrm{n}, \mathrm{k})$ code if and only if its 2 kcodewords form a $\mathrm{k}$ dimensional subspace of the vector space of all $n$-tuples over the Galois field GF. Since there are $\mathrm{n}$ output bits so there are $2 \mathrm{n}$ combinations possible for codewords but all of them are not codewords rather $2 \mathrm{k}$ are codewords. Rest of the combinations usually comes forward when there is an erroneous transmission and codewords are corrupted by change in bits. There are some rules which codewords have to fulfil. One of them is that the sum of any two codewords is also a codeword and being a linear vector space, there is some basis, and all codewords can be obtained as linear combinations of the basis [6]. Anexample of [5] code is generated by generator matrix given below:

$$
G=\left[\begin{array}{lllllll}
1 & 0 & 0 & 0 & 0 & 1 & 1 \\
0 & 1 & 0 & 0 & 1 & 0 & 1 \\
0 & 0 & 1 & 0 & 1 & 1 & 0 \\
0 & 0 & 0 & 1 & 1 & 1 & 1
\end{array}\right\rfloor
$$

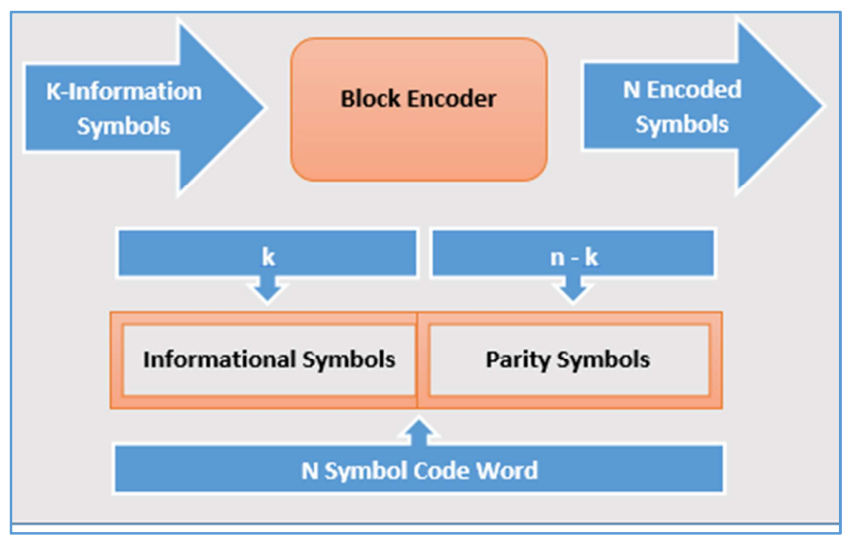

Figure 1. A block encoder and a LB coding codeword.

\subsection{Convolution Code}

Convolutional codes are different from the block codes [7], since in convolutional coding the information sequences are not grouped into distinct blocks and encoded so a continuous sequence of information bits is mapped into a continuous sequence of encoder output bits. Convolutional coding can achieve a larger coding gain than can be achieved using a block coding with the same code rate [8]. Convolutional codes have their popularity due to good performance and flexibility to achieve different coding rates.

An important characteristic of convolutional codes [9] is that the encoder has memory. That is the n-tuple output generated by the encoder is a function of not only the input $\mathrm{k}$-tuple but also the previous N-1 input k-tuples. The integer $\mathrm{N}$ is called the constraint length [7]. The convolutional code is generated by passing the information sequence through a finite state shift register. In general, the shift register contains $\mathrm{N}$ stages and $\mathrm{m}$ linear algebraic function generators based on the generator polynomials [9]. The input data $\mathrm{k}$ bits is shifted into and along the shift register. The number of output bits for each $\mathrm{k}$ input bits is $\mathrm{n}$ bits. The code rate is given as $\mathrm{R}=\mathrm{k} / \mathrm{n}$.

\subsection{RS Coding}

The mechanism by which Reed-Solomon codes [10] 
correct errors is that the encoder adds redundant bits to the digital input data block. The decoder attempts to correct and restore the original data by removing the errors that are introduced in transmission, due to many reasons that might be the noise in the channel or the scratches on a CD. There are different families of Reed-Solomon codes and each has its own abilities to correct the number and type of errors.

These codes are linear and a subsection of BCH codes [11] and can be denoted as $\mathrm{RS}(\mathrm{n}, \mathrm{k})$ with s-bit (Where $\mathrm{s}$ are symbols represented as a bit) symbols.

An n-symbol codeword is made by the k data symbols of $s$ bits each and the encoder adds parity bits. Errors in a codeword can be corrected by the decoder up to $t$ symbols where, $2 \mathrm{t}=\mathrm{n}-\mathrm{k}$.

\section{Research Methodology}

Quantitative research methodology (AI-Mahmoud \& Zoltowski, 2009) is adopted in this work. MATLAB is used as a tool to implement the OFDM system, error correcting techniques for the OFDM system and after that a performance comparison is made between these techniques. The outcomes of this work will be a BER (Bit Error Rate) vs. SNR (Signal to Noise Ratio) comparison, which will tell the behavior of all these three codes (Linear block codes, Convolutional codes and Reed-Solomon codes) under different SNR. The assessment will help us to analyze the performance of the three coding techniques in combination with OFDM. The finding of this work may help the OFDM system designers to choose the error correcting codes that match their requirements.

\subsection{Code Modification}

A code $\mathrm{C}$ has three fundamental parameters. Its length $\mathrm{n}$, its dimension $\mathrm{k}$, and its redundancy $\mathrm{r}=\mathrm{n}-\mathrm{k}$. Each of these parameters has a natural interpretation for linear codes, and although the six basic modification techniques [3], are not restricted to linear codes it will be easy initially to describe them in these terms. Each of these one parameter and increases or decreases the other two parameters accordingly. We have:

1. Augmenting. Fix n; increase k; decrease $r$

2. Expurgating. Fix n; decrease $\mathrm{k}$; increase $\mathrm{r}$

3. Extending. Fix k; increase $n$; increase $r$

4. Puncturing. Fix k; decrease $n$; decrease $r$

5. Lengthening. Fix r; increase $\mathrm{n}$; increase $\mathrm{k}$

6. Shortening. Fix r; decrease n; decrease k

The six techniques fall naturally into three pairs, each member of a pair the inverse process to the other. Since the redundancy of a code is its "dual dimension" each technique also has a natural dual technique. In this workwe used the extending feature of code modification. We have modified our OFDM input \& output code correction techniques according to the extended features and performed the simulation accordingly.

\subsection{Extending \& Puncturing}

In extending or puncturing [5] a code we keep its dimension fixed but vary its length and redundancy. These techniques are exceptional in that they are one-to-one. Issues related to the extending and puncturing of GRS codes will be discussed in the next two sections.

When extending a code we add extra redundancy symbols to it. The inverse is puncturing, in which we delete redundancy symbols. Puncturing may cause the minimum distance to decrease, but extending will not decrease the minimum distance and may, in fact, increase it. To extend a linear code we add columns to its generator matrix, and to puncture the code we delete columns from its generator.

Let us call the $[\mathrm{n}+1, \mathrm{k}]$ linear code $\mathrm{C}_{+}$a coordinate extension of $\mathrm{C}$ if it results from the addition of a single new redundancy symbol to the $[\mathrm{n}, \mathrm{k}]$ linear code $\mathrm{C}$ over the field F. Each codeword $c_{+}=\left(c_{1}, \ldots ., c_{n}, c_{n+1}\right)$ of the extended code $\mathrm{C}+$ is constructed by adding to the codeword $\mathrm{c}=\left(\mathrm{c}_{1}, \ldots \ldots, \mathrm{c}_{\mathrm{n}}\right)$ of $\mathrm{C}$ a new coordinate $C_{n+1}=\sum_{i=1}^{n} a_{i} c_{i}=a . c$, for some fixed $a=\left(a_{1}, \ldots \ldots, a_{n}\right) \in F_{n}$. Here $I$ imply that the new coordinate is the last one, but this is not necessary. A coordinate extension can add a new position at any place within the original code.

The most typical method of extending a code is the appending of an overall parity check symbol, a null symbol chosen so that the entries of each new codeword sum to zero. This corresponds to a coordinate extension in which ' $a$ ' has all of its entries equal to -1 .

\section{Simulation \& Results}

To keep the conditions same for all the simulations the input data, the fading channel and the noise is once generated and saved. The input data is kept same for testing of all coding schemes. The total number of carriers [12] chosen in the OFDM system is 128 and used is 104 . Eb/No (Energy per bit to noise power ratio) is used in testing. Eb/No is useful when comparing BER in digital modulation schemes without taking the bandwidth into account.

Rayleigh fading channel is once generated and saved. The same fading is used for all the simulations. The fading is defined by the following MATLAB equation:

$$
\text { rayleigh }=\operatorname{sqrt}(0.5 *(\operatorname{randn}(\mathrm{N}, 1)+\mathrm{i} * \operatorname{randn}(\mathrm{N}, 1)))
$$

Where 'randn()' generates values from the standard normal distribution function and ' $\mathrm{N}$ ' defines the length of fading signal.

The results of simulation for code rate of $1 / 3$ as per MATLAB simulation are shown in Figure 2. It is found that convolution code after the extending \& puncturing features applied gives $2 \mathrm{~dB}$ improvement than Reed Solomon Code and $4 \mathrm{~dB}$ improvement than flat Block Codes at BER of $10^{-3}$. 


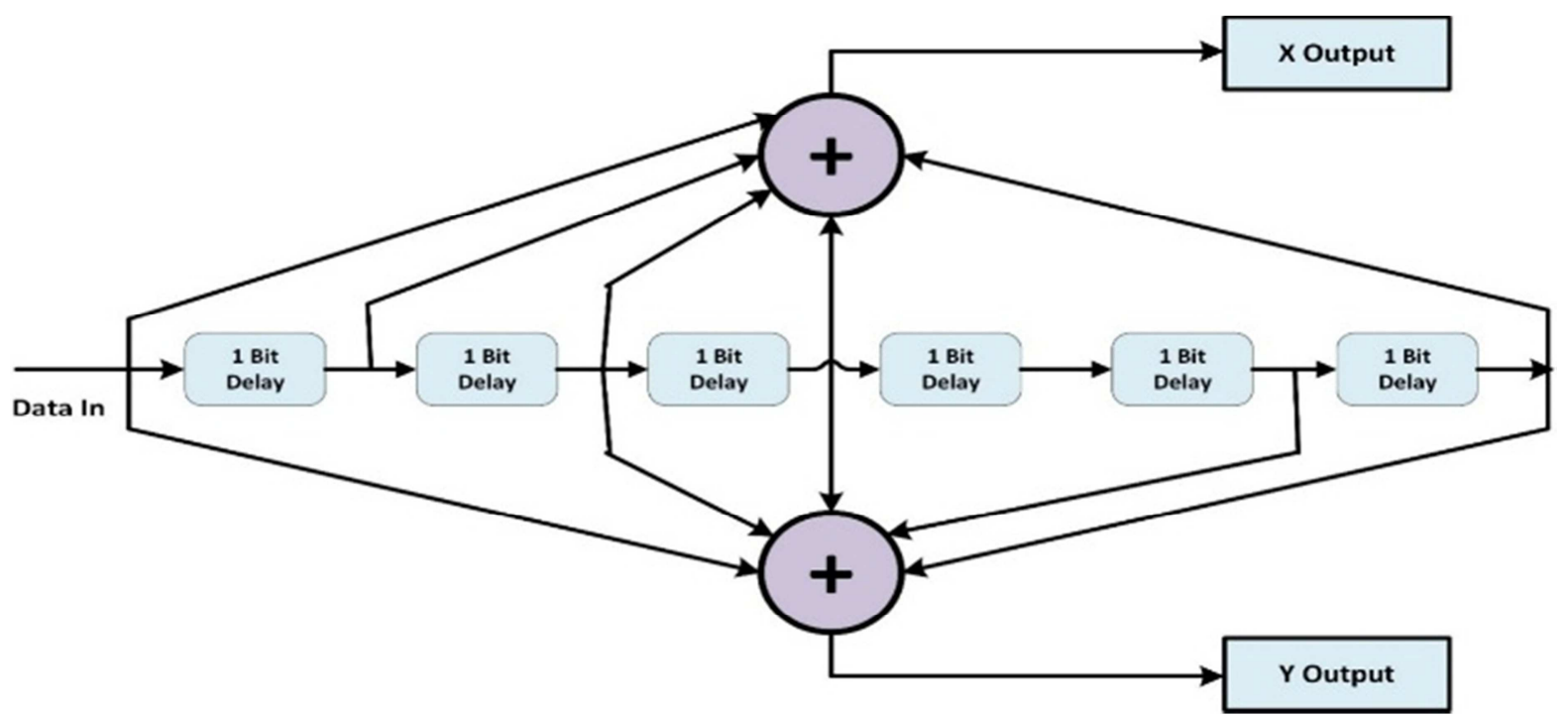

Figure 2. Convolutional encoder with length $7 \&$ rate $1 / 2$.

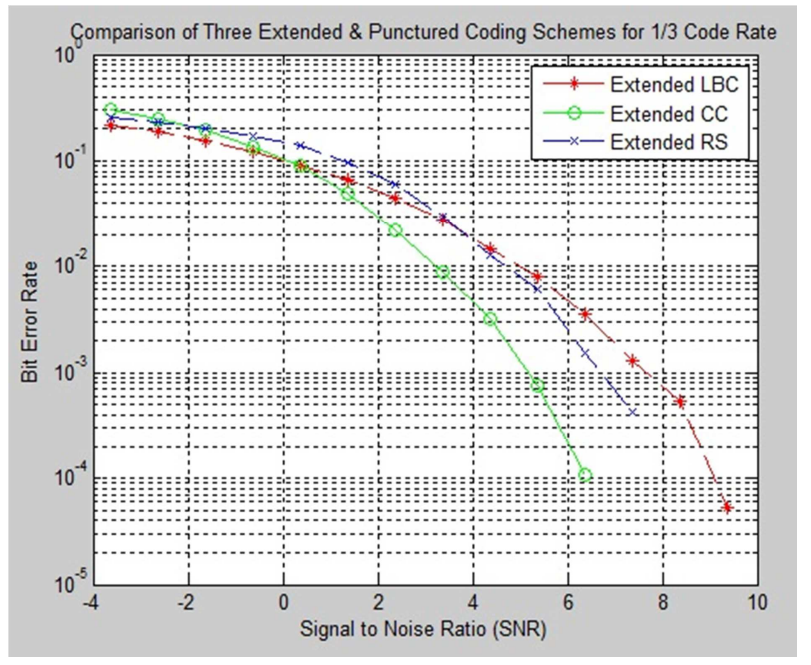

Figure 3. Comparison of Three Extended \& Punctured Coding Schemes for 1/3 Code Rate.



Figure 4. Comparison of Three Extended \& Punctured Coding Schemes for 1/2 Code Rate.
The results of simulation for code rate of $1 / 2$ are shown in Figure 3. It is found that RS code after the extending \& puncturing features applied gives $1 \mathrm{~dB}$ improvement than Convolution Code and $2 \mathrm{~dB}$ improvement than Block Codes at BER of $10^{-3}$.

The final results of simulation for code rate of $2 / 3$ are shown in Figure 4. It is found that Convolution code after the extending \& puncturing features applied gives. $5 \mathrm{~dB}$ improvement than RS Code and $1.5 \mathrm{~dB}$ improvement than Block Codes at BER of $10^{-3}$.



Figure 5. Comparison of Three Extended \& Punctured Coding Schemes for 2/3 Code Rate.

Hence from all the performance analysis made above for different scenarios are shown in the following graphs: 


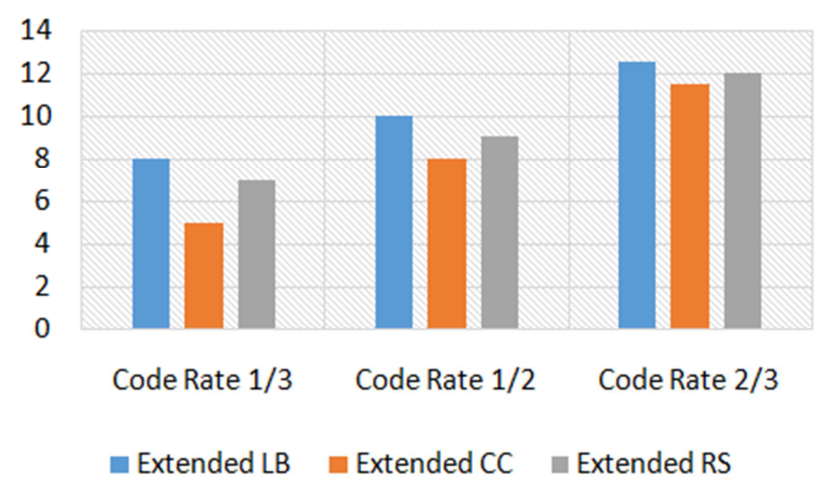

Figure 6. Comparison of all three error rectification techniques in terms of extended \& punctured coding scheme for OFDM systems.

\section{Conclusion}

In this work it is found that Convolutional codes are very good in performance at lower code rates but due to complex decoding structure they are difficult to implement. Another problem that may arise in them is error propagation while decoding. Convolutional codes have problem of very complex decoding so if the length of input data is increased, the trellis used will be complex and the decoding will become even more complicated.

Linear block codes are very simple to implement. They also show good performance and are also good for lower code rates and highly suitable for application with less complexity requirement and not so high performance requirement.

Reed-Solomon codes in comparison to linear block codes are difficult to implement but their performance is much better and consistent than linear block codes since they can handle long bursts of errors. These codes showed good performance on all three tested code rates.

Hence with the system that requires good performance and along with that can also handle complexity, there is no better than convolution code. But if the system can't handle complex architecture then RS codes might be more suitable for the purpose.

\section{Limitations}

Limitation of this work certainly includes the use of a model noise channel as this result is yet to be tested in physical condition. If someone changes the generator matrix then it will also change the results. Thus it is not possible to generalize these results by having different environment other than described in Section $\mathrm{V}$ in simulation environment.

\section{References}

[1] Prasad, R. (2000), OFDM for Wireless Communications Systems, Artech House Publishers, Boston, London.

[2] Hughes Software Systems (2002), Multi Carrier Code Division Multiple Access.

[3] Haykin, S. (2006), Communication Systems, John Wiley \& Sons, Inc.

[4] Sklar, B. (2001), Digital Communications: Fundamentals and Applications, Second Edition, Upper Saddle River, NJ: Prentice-Hall.

[5] Ryan, W. E., \& Lin, S. (2009), Channel Codes: Classical and Modern. Cambridge University Press.

[6] Agarwal, Arun, \& Patra, S. K. (2011). Performance Prediction of OFDM based DAB System using Block coding technique", Proceedings of ICETECT, India.

[7] MacKay, David J. C. (2003), Information Theory, Inference, and Learning Algorithms, Cambridge University Press.

[8] Witrisal, K., Kim, Y., \& Prasad, R. (1999), A Novel Approach for Performance Evaluation of OFDM with Error Correction Coding and Interleaving, Centre for Wireless Personal Communications (CEWPC), IRCTR Delft University of Technology.

[9] Chatzigeorgiou, I. A. (2012), A Comparison of Convolutional and Turbo Coding Schemes For Broadband FWA Systems.

[10] Koetter, R. (2005), Reed-Solomon Codes, MIT Lecture Notes.

[11] AI-Mahmoud, M., \& Zoltowski, Michael D. (2009), Performance Evaluation of CODE-SPREAD OFDM with error control coding, School of Electrical and Computer Engineering Purdue University West Lafayette, IN 479072035.

[12] Cho, Y. S., Kim, J. (2010), MIMO-OFDM Wireless Communications with MATLAB, John Wiley \& Sons, Inc.

[13] Haque, D., Enayet Ullah, S., \& Ahmed, R. (2008), Performance evaluation of a wireless Orthogonal Frequency Division Multiplexing system under various concatenated FEC channel-coding schemes, Proceedings of 11th International Conference on Computer and Information Technology, Khulna, Bangladesh, 25-27 December. 